\title{
Results of a patient survey for an implantable neurostimulator to treat migraine headaches
}

\author{
Koen Paemeleire · Amy M. Goodman
}

Received: 2 January 2012/ Accepted: 21 February 2012/Published online: 7 March 2012

(C) The Author(s) 2012. This article is published with open access at Springerlink.com

\begin{abstract}
Migraine attacks are believed to involve activation of the trigeminovascular system and trigeminalparasympathetic reflex, which is mediated through the sphenopalatine ganglion (SPG). An implantable SPG neurostimulator has been developed to apply on-demand SPG stimulation for the treatment of severe primary headache. The neurostimulator is implanted via an oral incision and placed along the maxilla, with the lead placed at the SPG. The neurostimulator contains no battery and is powered and controlled via a handheld remote controller. The potential interest of patients with high-frequency, highdisability migraine in having a SPG neurostimulator implanted to treat migraine is unknown. We aimed to evaluate patient interest to undergo such an implantation procedure and to participate in a clinical investigation of on-demand SPG stimulation for migraine by conducting a survey at the Ghent University Hospital in 41 migraineurs. Seventy-seven percent $(77 \%)$ of subjects expressed an interest in participating in a clinical investigation requiring implantation of a SPG neurostimulator when headache frequency and severity were considered and 69\% when pain relief experienced with current migraine treatment was considered. Preventive and acute medications were used in 64 and $95 \%$ of the subjects, respectively, and provided a reported reduction of headache frequency, duration and pain. However, acute medications were
\end{abstract}

\footnotetext{
K. Paemeleire $(\bowtie)$

Department of Neurology, Ghent University Hospital,

De Pintelaan 185, 9000 Ghent, Belgium

e-mail: koen.paemeleire@uzgent.be
}

\section{A. M. Goodman}

Clinical Affairs, Autonomic Technologies, Inc,

3698 Haven Avenue, Suite C, Redwood City, CA 94063, USA

e-mail: agoodman@autonomictechnologies.com frequently associated with headache recurrence and bothersome side effects. Results indicate that a majority of high-frequency, high-disability migraineurs, many of whom achieve pain relief with their current medications, have an interest in participating in a clinical investigation of an implantable SPG neurostimulator for the treatment of migraine headache.

Keywords Sphenopalatine ganglion (SPG) - Migraine · Headache $\cdot$ Neuromodulation $\cdot$ Neurostimulation

\section{Introduction}

The sphenopalatine ganglion (SPG) is an extracranial parasympathetic neural structure located in the pterygopalatine fossa. Migraine pain is believed to result from activation of the trigeminovascular system along with a trigeminal-parasympathetic reflex arc, which is mediated through the SPG [1, 2]. Cranial autonomic symptoms, which can be unilateral, are often associated with migraine headaches. These features are present in between 30 and $70 \%$ of migraine patients and include conjunctival injection, lacrimation, nasal congestion, and rhinorrhea [3]. These symptoms are the result of activation of the cranial parasympathetic system, and are believed to be due to activation of the trigeminal afferent arm of the trigeminalparasympathetic reflex [4].

SPG interventions have been used for over 100 years to treat headache pain [5]. These SPG procedures include pharmacological blocks [6], lesional and non-lesional ablations [7] and surgery [8]. Despite purposefully damaging or destroying the SPG as part of the therapy, results have been good with minimal side effects, although the procedures have not provided permanent headache relief. 
Therefore, the potential of electrical stimulation of the SPG has been explored in pilot studies for acute treatment of cluster and migraine headaches using a temporary electrode $[9,10]$. More recently, on-demand SPG stimulation for the treatment of chronic cluster headache has been evaluated in the Pathway $\mathrm{CH}-1$ study using a miniaturized neurostimulator developed by Autonomic Technologies, Inc. (ATI). The neurostimulator is implanted through a gingival buccal incision using standard oral surgery techniques and placed along the maxilla with the lead located at the SPG within the pterygopalatine fossa. Following implantation, a "titration" period allows for refinement of stimulation settings. During the experimental period, headaches were randomized to one of three stimulation doses, including full-, sub-perception and placebo stimulation.

The potential interest of patients with high-frequency, high-disability migraine in undergoing implantation of a SPG neurostimulator to treat migraine headache is unknown. A multi-center clinical investigation, the Pathway M-1 trial, is underway in Europe to evaluate both the acute and preventive effects of on-demand SPG stimulation. We aimed to evaluate patient interest in undergoing such an implantation procedure and participation in a clinical investigation of ondemand SPG stimulation for migraine.

\section{Methods}

A patient survey was conducted in 41 migraine patients at the Ghent University Hospital. Patients were required to have a MIDAS score of III to IV or a HIT-6 score greater than 56, to have 2-15 migraine pain days per month and to not have medication-overuse headache. Subjects were asked to consider their headache frequency and severity and their satisfaction with their current migraine treatment and to evaluate their willingness to undergo implantation of a neurostimulator for a clinical evaluation of SPG stimulation for migraine.

Patients were provided with general information regarding methods for using the ATI Neurostimulation System including that the neurostimulator is powered and controlled by holding a remote controller to the face (Fig. 1b). The implantation procedure was described as being similar to other types of oral surgery and requiring a few weeks to heal. Specific procedure-related adverse events were not provided. Specifically, patients were informed that the neurostimulator would be implanted through the mouth and placed behind the cheekbone (Fig. 1a), would not be visible after implant and would have no battery and thus would not require replacement, though if needed, could be removed using local anesthetic. The survey was designed to assess the percentage of attacks expected to be adequately treated in order for the patient to consider participation in a clinical investigation,
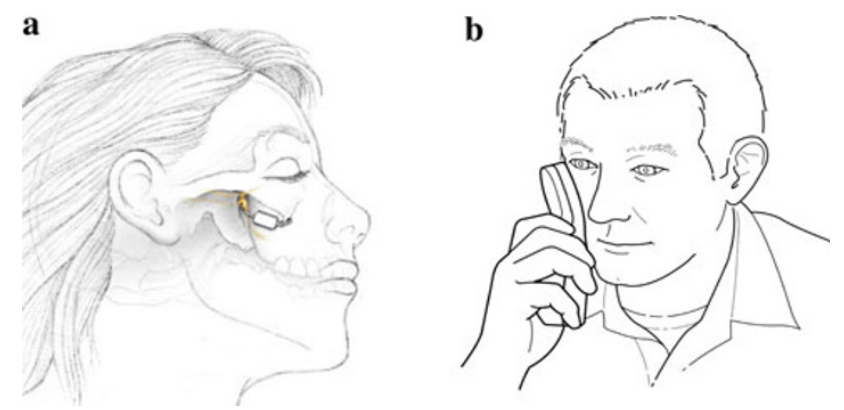

Fig. 1 a Diagram showing the location of the implant behind the cheekbone. b Diagram showing how the remote controller is held over the cheek to control the activation of the neurostimulator

thus, information regarding expectations for pain relief was not provided.

\section{Results}

The subject population (Tables 1,2) for the survey consisted largely of female high-frequency, high-disability episodic migraineurs with a high percentage of associated migraine symptoms. Subjects were recruited from the Outpatient Neurology Clinic of the Ghent University Hospital. Of the 41 subjects surveyed, 64\% currently used preventive medication for their migraines, which were reported to reduce both migraine frequency $(65 \%)$ and duration of the attacks (52\%), but caused bothersome side effects in some subjects (24\%). Thirty-nine subjects (95\%) used acute medications which provided pain relief $(75 \%)$ and pain freedom (68\%), but were frequently associated with both headache recurrence (93\%) and bothersome side effects $(69 \%)$.

Table 1 Subject population

\begin{tabular}{ll}
\hline Gender & Female 79\% \\
Age & 37 (range 18-76) \\
Years of migraines & 17 (range 2-66) \\
Frequency & Attacks/month: 7 (range 2-15) \\
& Days/month: 11 (range 4-20) \\
& $<1$ day: $9 \%$ \\
Duration & $1-2$ days: 55\% \\
& 2 days: $36 \%$ \\
Symptoms & Nausea/vomiting: 66\% \\
& Light sensitivity: $90 \%$ \\
& Sound sensitivity: $76 \%$ \\
Disability scores & MIDAS \\
& I: 2 III: 13 \\
& II: 0 IV: 26 \\
& HIT-6 \\
& 64 (range 57-72)
\end{tabular}


Table 2 Preventive and acute medication usage

\begin{tabular}{ll}
\hline Preventive medication usage & Acute medication usage \\
\hline $64 \%$ Currently taking & $95 \%$ Currently taking \\
preventive medication & acute medications \\
$65 \%$ Reduction in migraine frequency & $75 \%$ Pain reduction \\
$52 \%$ Reduction in headache duration & $68 \%$ Pain freedom \\
$24 \%$ Bothersome side effects & $93 \%$ Migraine headache \\
& recurrence \\
& $69 \%$ Bothersome side effects
\end{tabular}

The majority of subjects used both preventive and acute medications. Although these medications were reported to reduce migraine frequency, duration and pain, the majority of subjects indicated they would consider participating in an investigation of a SPG neurostimulator for migraine. Specifically, given headache frequency/severity, and given the pain relief experienced with current migraine treatment, 77 and $69 \%$ of subjects, respectively, indicated a willingness to consider participating in a clinical investigation requiring implantation of a SPG neurostimulator. Of the subjects who would consider receiving an implant and participating in the study, $100 \%$ expected the therapy to treat at least $50 \%$, and $81 \%$ expected the therapy to treat at least $75 \%$ of their migraines. Thus, the survey indicated an expectation that an implanted device to treat migraine should treat a majority of migraine attacks.

\section{Conclusion}

Sphenopalatine ganglion stimulation using an implanted, ondemand neurostimulator is a novel, promising therapy option for migraine sufferers, although the ability of SPG stimulation to provide acute relief of migraine pain has not yet been demonstrated. Results of the survey conducted at the Ghent University Hospital indicate that a majority of high-frequency, high-disability migraineurs, many of whom achieve pain relief with their current medications, are willing to undergo implantation of a SPG neurostimulator and participate in a clinical investigation of SPG stimulation for the treatment of migraine headache, expecting a very efficacious non-pharmacological therapy alternative. Patient expectations regarding pain relief should be assessed and discussed prior to enrollment in the study. Detailed information on potential side effects and adverse events will further influence a patient's decision to participate, but was not included in this survey.

Acknowledgments The survey was funded with a research grant provided by Autonomic Technologies Inc, Redwood City, CA, USA.

Conflict of interest Koen Paemeleire has received fees for serving as a speaker, an advisory board member or participation in clinical studies from Allergan, Almirall, Autonomic Technologies Inc, Coherex, Janssen-Cilag, Medtronic, Pfizer, Sandoz, and St Jude Medical. Amy M. Goodman is an employee of Autonomic Technologies, Inc.

Open Access This article is distributed under the terms of the Creative Commons Attribution License which permits any use, distribution, and reproduction in any medium, provided the original author(s) and the source are credited.

\section{References}

1. Goadsby PJ, Lipton RB, Ferrari MD (2002) Migraine-current understanding and treatment. N Engl J Med 346(4):257-270

2. Yarnitsky D, Goor-Aryeh I, Bajwa ZH, Ransil BI, Cutrer FM, Sottile A, Burstein R (2003) 2003 Wolff Award: possible parasympathetic contributions to peripheral and central sensitization during migraine. Headache 43(7):704-714

3. Barbanti P, Fabbrini G, Pesare M, Vanacore N, Cerbo R (2002) Unilateral cranial autonomic symptoms in migraine. Cephalalgia 22(4):256-259

4. May A, Goadsby PJ (1999) The trigeminovascular system in humans: pathophysiologic implications for primary headache syndromes of the neural influences on the cerebral circulation. J Cereb Blood Flow Metab 19(2):115-127

5. Sluder G (1908) The role of the sphenopalatine (or Meckle's) ganglion in nasal headaches. New York Med J 87:989-990

6. Maizels M, Scott B, Cohen W, Chen W (1996) Intranasal lidocaine for treatment of migraine: a randomized, double-blind, controlled trial. JAMA 276(4):319-321

7. Narouze S, Kapural L, Casanova J, Mekhail N (2009) Sphenopalatine ganglion radiofrequency ablation for the management of chronic cluster headache. Headache 49(4):571-577

8. Meyer JS, Binns PM, Ericsson AD, Vulpe M (1970) Sphenopalatine ganglionectomy for cluster headache. Arch Otolaryngol 92(5):475-484

9. Ansarinia M, Rezai A, Tepper SJ, Steiner CP, Stump J, StantonHicks M, Machado A, Narouze S (2010) Electrical stimulation of sphenopalatine ganglion for acute treatment of cluster headaches. Headache 50(7):1164-1174

10. Tepper SJ, Rezai A, Narouze S, Steiner C, Mohajer P, Ansarinia M (2009) Acute treatment of intractable migraine with sphenopalatine ganglion electrical stimulation. Headache 49(7):983-989 